\title{
A Novel Strategy for Improving the Quality of Embedded Zerotree Wavelet Images Transmitted over Alamouti Coding Systems
}

\author{
Josmary Labrador, Paula M. Castro, \\ Héctor J. Pérez-Iglesias, and Adriana Dapena \\ Department of Electronics and Systems, University of A Coruña, \\ Campus de Elviña s/n, 15.071. A Coruña. Spain. \\ \{jlabrador, pcastro, hperez, adriana\}@udc.es
}

\begin{abstract}
This work deals with the transmission of images, previously coded using the Embedded Zerotree Wavelet (EZW) transform, over wireless systems in which Space-Time Coding (STC) is used. It is shown how the system performance, measured in terms of Peak Signal to Noise Ratio (PSNR), can be improved using bit allocation strategies that take into account the special structure of the EZW bitstream, where the bits firstly allocated are associated to the lowest frequency subbands, and therefore, an error-free transmission of such bits will be crucial to appropriately recover the transmitted image.
\end{abstract}

Keywords: Artificial neural networks, learning rules, EZW transform, Alamouti coding, PSNR metric, image processing, bit allocation, channel estimation.

\section{Introduction}

The Embedded Zerotree Wavelet (EZW) transform is a quite simple image compression algorithm based on a tree-ordering of the wavelet coefficients $[1,2]$. By taking into account that, for wavelet transforms, the highest energy coefficients reside in the lowest frequency subbands placed at the root node, and that a parent-child relationship is defined between wavelet coefficients from frequency subbands spatially related, so that the children correspond to higher frequencies than their respective parents, one or more subtrees will entirely have coefficients whose value is zero or almost zero with high probability. Those subtrees are called zerotrees. Therefore, the bitstream is organized according to the wavelet coefficients ordered from lowest to highest frequency subbands, which allows the decoder to stop the decoding process at any point of the bitstream and still recover the transmitted images but with lower quality. This property is termed as progressive or incremental decoding.

This paper focuses on the transmission of EZW-coded images over wireless systems making use of the popular Orthogonal Space-Time Block Code (OSTBC) scheme proposed by Alamouti [4], which has been incorporated to the 
IEEE 802.11 and IEEE 802.16 standards, for example. Coherent detection using Alamouti-coded systems demands a unitary channel matrix, which is commonly acquired from transmitted training symbols (pilots) by means of supervised algorithms $[5,6]$. In order to increase the system throughput, there exists a great interest about the development of algorithms to directly estimate the channel from the observations without using pilots. This type of algorithms are termed as unsupervised or blind [7].

Principal Component Analysis (PCA) was developed in 1901 by Karl Pearson and it is one of the most important paradigms in Artificial Neural Networks (ANN) since it is connected with Hebbian-type learning rules [8]. Nowadays, PCA is mostly used as a tool for data analysis and for predictive modeling. Shahbazpanahi et al. in [9] have shown that the channel matrix of Alamouti coding systems can be estimated using PCA although it requires different energies for signal transmission. This unbalanced energy implies a degradation in terms of Bit Error Rate (BER) for the signal transmitted with lower energy. In fact, for EZW-coded images it will be shown how Peak Signal to Noise Ratio (PSNR) can be considerably improved when the bits associated to the lowest frequency subbands are sent using higher energy than that assigned for the bits corresponding to higher subbands. However, this bit allocation strategy penalizes the EZW property for incremental decoding and, for this reason, it is also proposed in this work a simple decision criterion to decide when that strategy must be used or not.

The work is structured as follows. The channel model is shown in Section 2, where the utilization of PCA for channel estimation is also described. Section 3 shows three different strategies to convert the EZW bitstream to the signals transmitted through the antennas, and Section 4 compares those strategies by performing several computer simulations. Finally, Section 5 presents the main work conclusions.

\section{Alamouti Coding Systems}

We define the sources $s_{1}$ and $s_{2}$ as independent equiprobable discrete random variables with values from a finite set of symbols belonging to a real or complex modulation (PAM, PSK, QAM...). In a $2 \times 1$ Multiple-Input/Single-Output (MISO) case, the vector $\mathbf{x}=\left[\begin{array}{ll}x_{1} & x_{2}\end{array}\right]^{T}$ of received signals (so-called observations) can be written as $\mathbf{x}=\mathbf{H s}+\mathbf{v}$, where $\mathbf{s}=\left[\begin{array}{ll}s_{1} & s_{2}\end{array}\right]^{T}$ is the source vector, $\mathbf{v}=\left[\begin{array}{ll}v_{1} & v_{2}\end{array}\right]^{T}$ is the Additive White Gaussian Noise (AWGN) vector, and the $2 \times 2$ channel matrix has the form

$$
\mathbf{H}=\left[\begin{array}{rr}
h_{1} & h_{2} \\
h_{2}^{*} & -h_{1}^{*}
\end{array}\right] .
$$

Note that this matrix is orthogonal, i.e. $\mathbf{H}^{\mathrm{H}} \mathbf{H}=\mathbf{H H}^{\mathrm{H}}=\mathbf{I}_{2}\|\mathbf{h}\|_{2}^{2}$, where $\|\mathbf{h}\|_{2}^{2}=$ $\left|h_{1}\right|^{2}+\left|h_{2}\right|^{2}$ and thus, the sources can be recovered applying $\hat{\mathbf{s}}=\mathbf{H}^{\mathrm{H}} \mathbf{x}$.

In recent years, several unsupervised methods to estimate the channel matrix (and to recover the sources) have been developed assuming that both sources 
and channel matrices are completely unknown at the receiver side (see, for instance, [10] and references therein). PCA constitutes an interesting unsupervised method to estimate the channel matrix since it implies to perform a reduced number of operations. By considering the covariance matrix $\mathbf{C}_{\mathbf{x}}$ obtained from the observations, i.e. $\mathbf{C}_{\mathbf{x}}=\mathrm{E}\left[\mathbf{x x}^{\mathrm{H}}\right], \mathrm{PCA}$ computes an orthogonal basis $\mathbf{U}$, so that a new set of orthogonal signals, given by $\mathbf{z}=\mathbf{U}^{H} \mathbf{x}$, can be obtained [8].

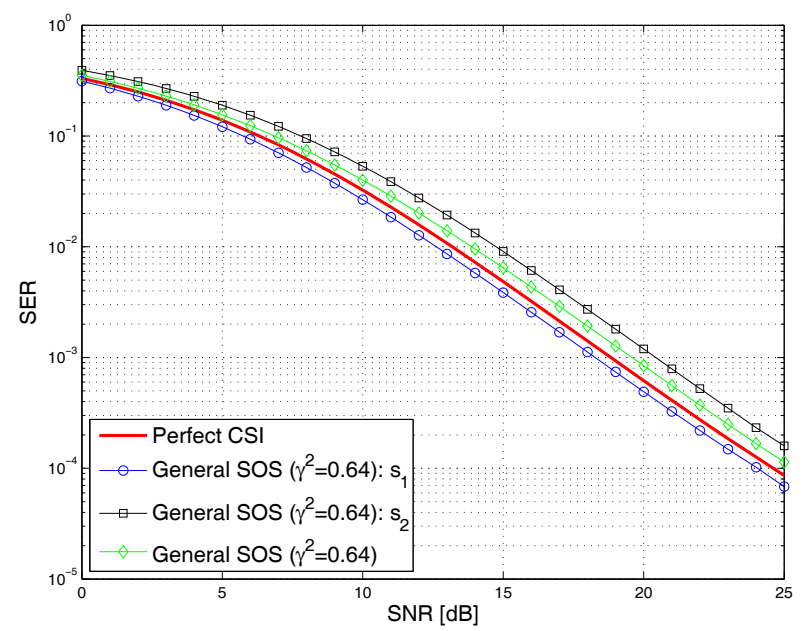

Fig. 1. SER performance of general SOS algorithm for randomly generated symbols.

For the scenario of Alamouti coding systems, since $\mathbf{H}$ is unitary, the matrix $\mathbf{U}$ is simply a normalized version of the channel matrix $\mathbf{H}$. It has been proved in [9] that the identification of the channel matrix is possible only when the sources have different energies, i.e. when the source $s_{1}$ is transmitted with an energy given by $E\left[\left|s_{1}(n)\right|^{2}\right]=2 /\left(1+\gamma^{2}\right)$, while $s_{2}$ is sent with the energy $E\left[\left|s_{2}(n)\right|^{2}\right]=$ $2 \gamma^{2} /\left(1+\gamma^{2}\right)$, where $\gamma$ is the parameter of energy unbalance.

Many methods can be applied to compute the PCA decomposition. For instance, Via et al. in [11] have proposed an adaptive learning procedure, while Pérez et al. in [10] have presented a block algorithm for that purpose. In both cases, the matrix $\mathbf{C}_{\mathbf{x}}$ is estimated by sampling averaging of the $N_{B}$ symbols received per frame.

In order to illustrate the degradation associated to the source energy unbalance, we consider a scenario where blocks of $N_{B}=1000$ symbols are generated from an equiprobable distribution. These symbols are modulated using 4-QAM and transmitted through block fading Rayleigh channels. We use the PCA implementation presented in [10] referred to as general Sum-Of-Square (SOS) reconstruction, where the aforementioned unbalancing parameter, $\gamma$, is used and whose value is set up to $\gamma^{2}=0.64$, i.e. $\gamma=0.8$. Figure 1 plots the Symbol Error Rate (SER) in terms of Signal Noise Ratio (SNR) for each source, $s_{1}$ and $s_{2}$, and the corresponding mean SER value. As a reference, it is also depicted SER under 
Perfect Channel Side Information (Perfect CSI) assumptions. Note that Perfect CSI corresponds to the mean SER obtained for the two sources $\left(s_{1}\right.$ and $\left.s_{2}\right)$ when CSI is perfectly known at the receiver side. By comparing the perfect CSI curve to those obtained using PCA, it can be seen from the figure that the source with highest energy $\left(s_{1}\right)$ exhibits lower SER, while the source with smaller one $\left(s_{2}\right)$ suffers from a loss in terms of SER with respect to perfect CSI scenario.

\section{Bit Allocation Strategies}

The results above reported show that the unbalanced energy is an important drawback for the use of PCA because it produces a SER degradation for one of the sources. In this section, we show that this degradation can be compensated with an adequate bit allocation taking into account the bit organization into the stream. The common strategy to convert a bitstream to a modulated signal (like, for example, an M-QAM signal), consists of sequentially processing that bitstream by taking groups of $b=\log _{2}(M)$ bits, which are mapped to their corresponding modulated symbol. The modulated symbols are subsequently divided into two sources (remember that a $2 \times 1$ Alamouti coded system is implemented): odd symbols, which are sent by the source $s_{1}$, and even symbols, which are sent by $s_{2}$. For EZW, such bit allocation implies that bits associated to any frequency subband are transmitted by both sources and, as a consequence, the degradation caused by unbalanced energy affects to all the subbands. This bit allocation is termed as Approach 1 in the following.

On the other hand, as a result of the structure observed for the EZW bitstream, we propose a novel bit allocation strategy (termed as Approach 2 in this work), where the bitstream is divided into two parts. The first part (corresponding to the lower subbands) is transmitted by $s_{1}$, while the second part (corresponding to higher subbands and to the so-called refinement bits) by $s_{2}$. This method permits that the energy degradation does not affect to the lower subbands but note, however, that it keeps from the incremental decoding of EZW. For this reason, it is desirable to use this approach only when the resulting quality of the recovered image is considerably better than that obtained applying Approach 1.

The immense majority of current wireless communication standards make use of feedback channels (usually limited in terms of throughput) between both sides of the link to periodically send channel state information from the receiver to the transmitter. For example, Worldwide Interoperability for Microwave Access (WiMAX) standard uses this channel to send an index for selecting the most adequate code according to channel conditions. In this work, we propose to use this feedback channel to indicate to the transmitter which bit allocation strategy must be used in order to obtain an adequate PSNR.

Since it is not possible to compute the PSNR at the receiver-end for a given image, it is also proposed to use a set of training images to get the estimated PSNR according to visual image quality as a function of SNR. Then, this information is stored at the receiver. Before the transmission of each new image, the 

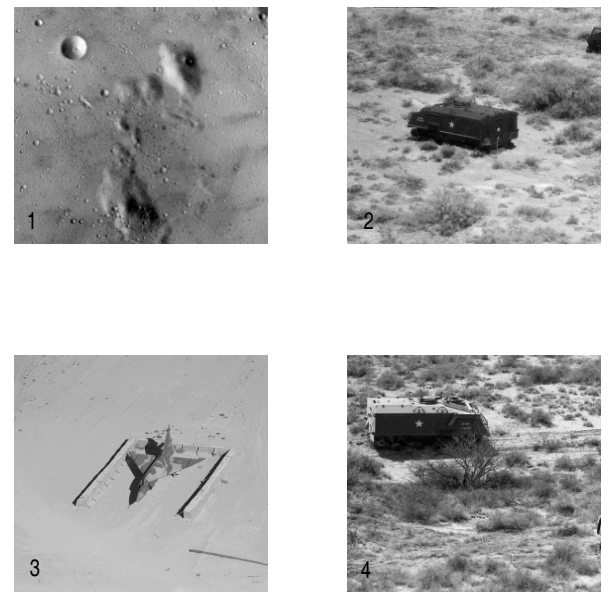

Fig. 2. Computer simulations: Training images.

receiver estimates the SNR and decides the bit allocation approach with higher estimated PSNR for a given SNR. An alternative way of interpreting this rule consists of defining the SNR threshold, denoted by $\mathrm{SNR}_{t}$, which marks out the working regions for each approach. In other words, this approach, termed as Hybrid Approach, can be described by the following decision rule

$$
\begin{gathered}
\text { estimated } \mathrm{SNR} \geq \mathrm{SNR}_{\mathrm{t}} \rightarrow \text { Use Approach } 1 \\
\text { estimated } \mathrm{SNR}<\mathrm{SNR}_{\mathrm{t}} \rightarrow \text { Use Approach 2, }
\end{gathered}
$$

whose result is sent to the transmitter through the feedback channel. The open issue is how to find that SNR threshold, $\mathrm{SNR}_{\mathrm{t}}$, which defines the border between the two working regions. In the next section, we will show a method based on the visual quality of the recovered images.

\section{Computer Simulations}

In order to compare the proposed bit allocation strategies, we consider a computer scenario where the bitstream of EZW-coded images is modulated using 4-QAM. The symbols are transmitted in blocks of size $N_{B}=1000$ using Alamouti coding. In order to guarantee that the channel matrix can be estimated using PCA, the transmitter unbalances the source energy by means of a parameter $\gamma^{2}=0.64$. Thus, PCA is used to acquire the channel matrix estimate per received frame. Note that the covariance matrix obtained from the observations is computed using all the frame symbols, i.e. $N_{B}$ symbols.

For the training step, the four images plotted in Figure 2 have been coded using EZW ${ }^{1}$. Figure 3 plots the PSNR in terms of SNR obtained by averaging

\footnotetext{
$\overline{1}$ The original images are constituted by $256 \times 256$ pixels with 256 gray levels.
} 

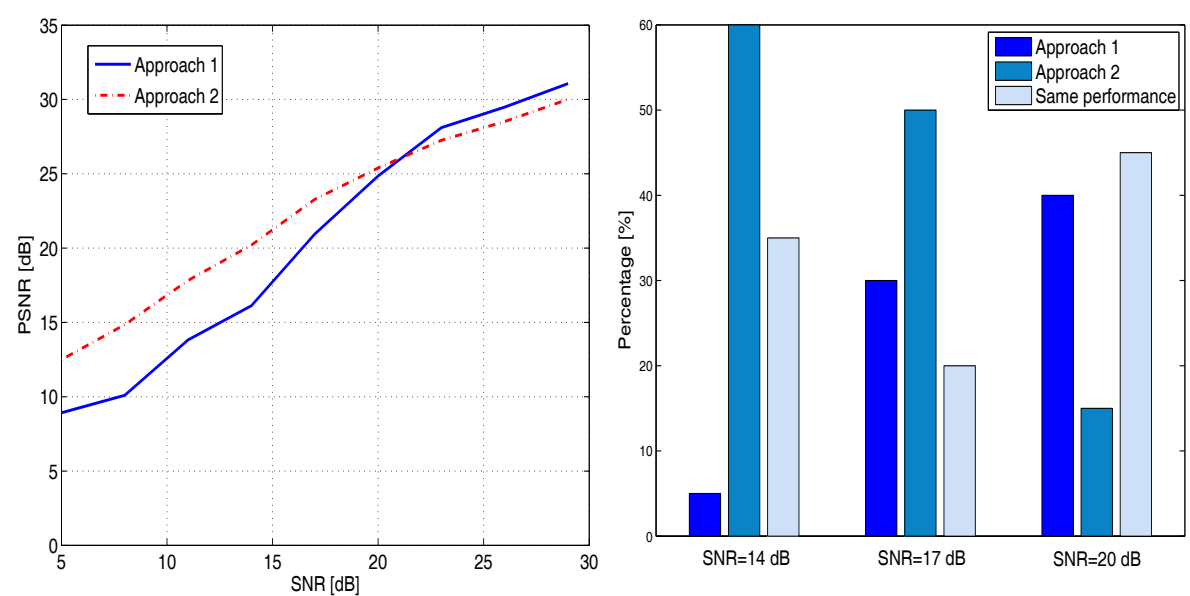

Fig. 3. Training step: Establishment of $\mathrm{SNR}_{\mathrm{t}}$ threshold using visual test.

the results for 10 Rayleigh channels randomly generated. It can be seen from this figure that the Approach 2 provides the best PSNR for low and medium SNR values since bits associated to lower frequency subbands are sent by means of the source having highest energy. With the goal of establishing the threshold parameter $\mathrm{SNR}_{\mathrm{t}}$ for the decision rule described in Equation (2), the recovered images corresponding to different SNR values, specifically 14,17 , and $20 \mathrm{~dB}$ for 10 channel realizations, have been empirically observed. A test oriented to decide which approach provides the best quality percentage or ratio taking into account all the set of training images is applied. Thus, Figure 3 also shows the results obtained from this visual test, which allows us to conclude that

- Firstly, for an SNR value of $14 \mathrm{~dB}$, the improvement achieved with Approach 2 compared to Approach 1 is substantial. Therefore, it is apparent that Approach 2 is the best choice in such a case leading to better quality reconstruction.

- Secondly, for an SNR value of $17 \mathrm{~dB}$, the improvement achieved with Approach 2 compared to Approach 1 is not as significant. By considering the results obtained with Approach 1 and that both approaches exhibit exactly the same performance for about a ratio of 20 to 100 (this scenario is labeled in the figure as Same performance), Approach 1 can be decided as a more adequate choice than Approach 2 if progressive decoding is desired.

- Finally, for an SNR value of $20 \mathrm{~dB}$, Approach 1 is clearly the best choice, since it is only outperformed by Approach 2 less than $20 \%$ of cases.

As a result, it can be established a threshold parameter of SNR equal to $17 \mathrm{~dB}$.

Figure 4 shows a comparison in terms of PSNR versus SNR between the three bit allocation strategies studied in this work only considering one of the test images depicted in Figure 2 and 10 channel realizations. Figure 5 illustrates 
the visual quality of the recovered images corresponding to SNR values of 14,17 , and $20 \mathrm{~dB}$ for a given channel realization, where the black rectangle marks the recovered ones in the event of implementing the above proposed hybrid approach.

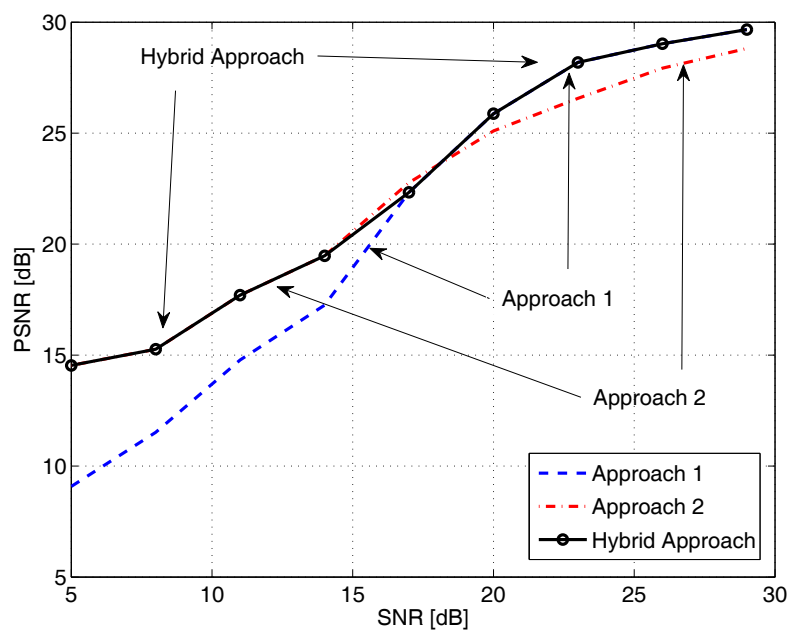

Fig. 4. PSNR results obtained from computer simulations for image 1.

\section{Conclusions and Future Work}

A lot of algorithms based on wavelets for image compressing, like EZW, produce bitstreams by ordering the subbands from lowest to highest, which allows to achieve different image qualities after the progressive decoder. This means that the first bits of the EZW sequence are "more important" than the following bits. In this work, we have used this property to improve the performance of the EZW images transmitted over wireless systems where Alamouti space-time block coding is used. We have focused on the utilization of PCA to acquire the channel matrix estimate avoiding the use of pilot symbols. As a result, three proposed bit allocation strategies combined with a decision rule based on an empirical SNR threshold parameter allow us to improve the quality of the reconstructed images after their transmission affected by fluctuating wireless channel conditions.

Future work to be developed by the authors is focused on studying different bit allocation strategies like, for instance, the sending of the refinement bits corresponding to LL subband through the channel with largest energy. Since PSNR criterion above explained is empirically obtained, other assessment metrics must be analyzed, specially those based on Human Visual System, as for example the Visual Information Fidelity measure. Additionally, this work could be extended to the standard of image processing JPEG2000, thus avoiding some of the drawbacks inherent to EZW. 


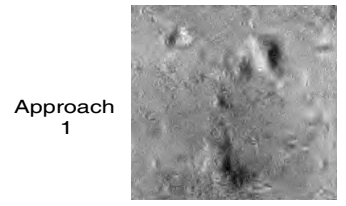

$\mathrm{SNR}=14 \mathrm{~dB}$

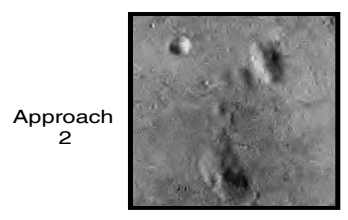

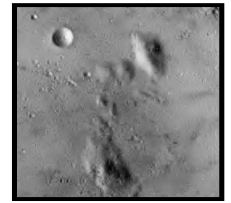

$\mathrm{SNR}=17 \mathrm{~dB}$

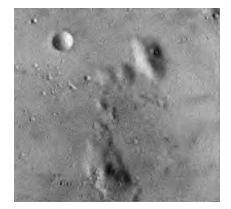

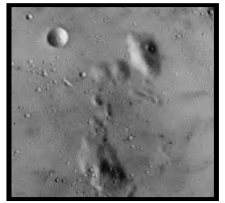

$\mathrm{SNR}=20 \mathrm{~dB}$

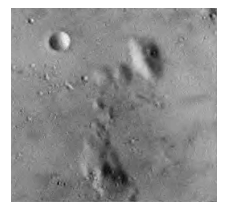

Fig. 5. Black rectangles indicate the reconstructed images for each SNR.

Acknowledgments. This work was supported by Xunta de Galicia (grant numbers 10TIC105003PR and 09TIC008105PR ) and Ministerio de Ciencia e Innovación of Spain (grant numbers TEC2010-19545-C04-01 and CSD2008-00010).

\section{References}

1. Taubman, D. S., Marcellin, M. W.: JPEG2000 Image Compression Fundamentals, Standards and Practice. Kluwer Academic Publishers (2002)

2. Shapiro, J. M.: Embedded image coding using zerotrees of wavelet coefficients. In: IEEE Transactions on Signal Processing, vol. 41, no. 12, pp. 3445-3462 (1993)

3. Shapiro, J. M.: An embedded hierarchical image coder using zerotrees of wavelet coefficients. In: Proc. of IEEE Data Compression Conference, pp. 214-223 (1993)

4. Alamouti, S. M.: A Simple Transmit Diversity Technique for Wireless Communications. In: IEEE Journal Select. Areas Communications, vol. 16, pp. 1451-1458 (1998)

5. Budianu, C., Tong, L.: Channel Estimation for Space-Time Orthogonal Block Codes. In: Proc. of International Conference on communications, pp. 1127-1131 (2001)

6. Naguib, A. F., Tarokh, V., Seshadri, N., Calderbank, A. R.: A Space-Time Coding Modem for High-Data-Rate Wireless. In: IEEE Journal on Selected Areas in Communications, vol. 16, no. 8, pp. 1459-1478 (1998)

7. Comon, P., Jutten, C.: Handbook of Blind Source Separation, Independent Component Analysis and Applications. Academic Press (2010)

8. Haykin, S.: Neural Networks: A Comprehensive Foundation. MacMillan Publishing Company (1994)

9. Shahbazpanahi, S., Gershman, A. B., Manton, J.: Closed-form Blind MIMO Channel Estimation for Orthogonal Space-Time Block Codes. In: IEEE Trans. on Signal Processing, vol. 53, no. 12, pp. 4506-4516 (2005)

10. Pérez-Iglesias, H. J., García-Naya, J. A., Dapena, A., Castedo, L., Zarzoso, V.: Blind Channel Identification in Alamouti Coded Systems: A Comparative Study of Eigendecomposition Methods in Indoor Transmissions at 2.4 GHz. In: European Transactions on Telecomunications (Special Issue), vol. 19, Issue 7, pp. 751-759, (2008)

11. Vía, J., Santamaría, I., Pérez, J., Ramírez, D.: Blind Decoding of MISO-OSTBC Systems based on Principal Component Analysis. In: Proc. of International Conference on Acoustic, Speech and Signal Processing, vol. IV, pp. 545-549 (2006) 